This item was submitted to Loughborough's Research Repository by the author.

Items in Figshare are protected by copyright, with all rights reserved, unless otherwise indicated.

\title{
Microstructural analysis of non-woven fabrics using scanning electron microscopy and image processing. Part 2: application to hydroentangled fabrics
}

\section{PLEASE CITE THE PUBLISHED VERSION}

\section{PUBLISHER}

(C) Professional Engineering Publishing

\section{VERSION}

VoR (Version of Record)

LICENCE

CC BY-NC-ND 4.0

\section{REPOSITORY RECORD}

Ghassemieh, E., Memis Acar, and Hendrik K. Versteeg. 2019. "Microstructural Analysis of Non-woven Fabrics Using Scanning Electron Microscopy and Image Processing. Part 2: Application to Hydroentangled Fabrics". figshare. https://hdl.handle.net/2134/4516. 
This item was submitted to Loughborough's Institutional Repository (https://dspace.lboro.ac.uk/) by the author and is made available under the following Creative Commons Licence conditions.

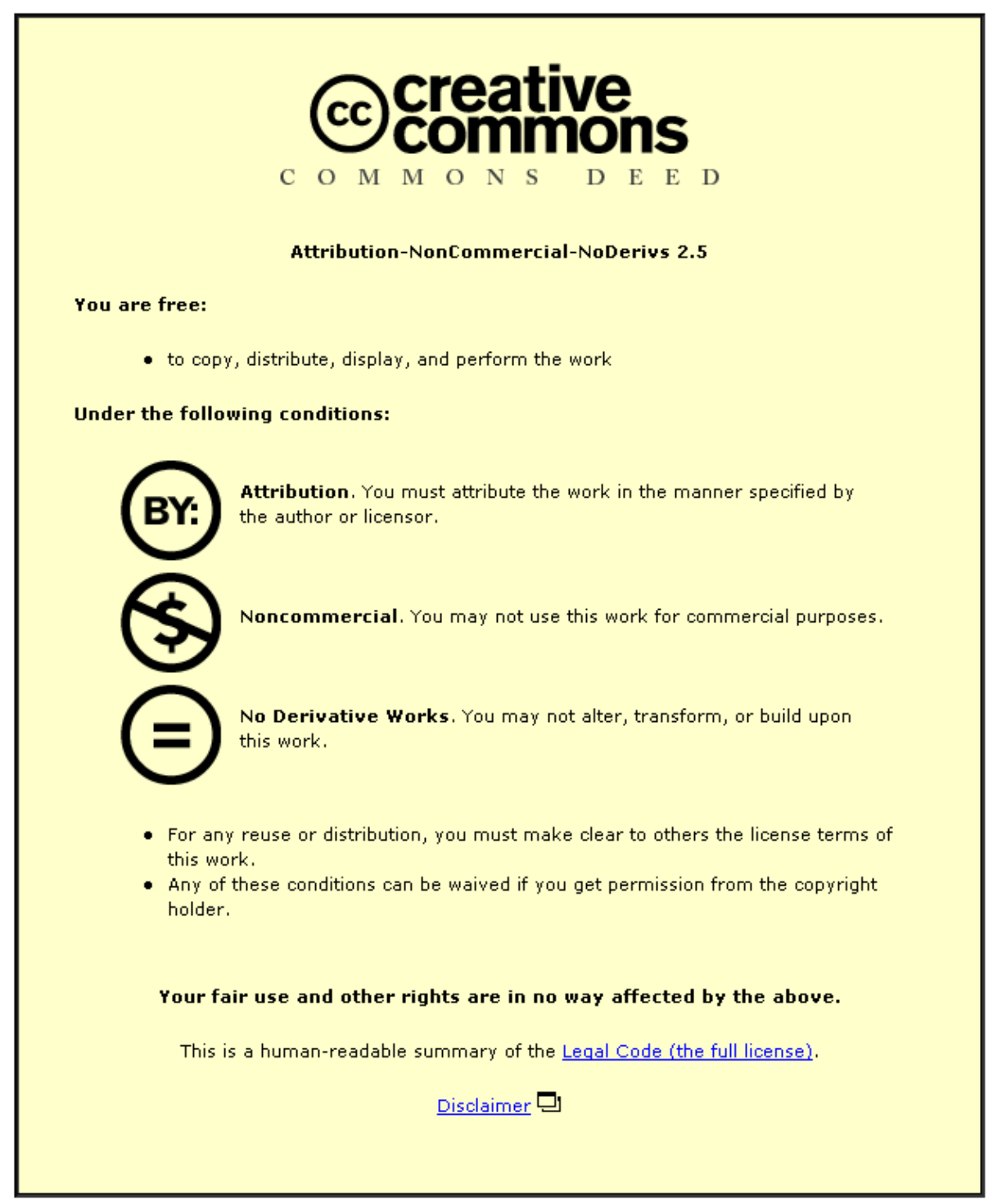

For the full text of this licence, please go to: http://creativecommons.org/licenses/by-nc-nd/2.5/ 


\title{
Microstructural analysis of non-woven fabrics using scanning electron microscopy and image processing. Part 2: application to hydroentangled fabrics
}

\author{
E Ghassemieh*, M Acar and H K Versteeg \\ Mechanical and Manufacturing Engineering, Loughborough University, Leicestershire, UK
}

\begin{abstract}
The image analysis techniques developed in Part 1 to study microstructural changes in nonwoven fabrics are applied to measure the fibre orientation distribution and fibre length distribution of hydroentangled fabrics. The results are supported by strength and modulus measurements using samples from the same fabrics. It is shown that the techniques developed can successfully be used to assess the degree of entanglement of hydroentangled fabrics regardless of their thickness.
\end{abstract}

Keywords: non-woven, fast Fourier transform, Hough transform, microstructure, scanning electron microscope

\section{INTRODUCTION}

The mechanical properties of non-woven fabrics and fibrous assemblies depend greatly on their microstructure. The arrangement of the fibres in the assembly to a large extend determines the mechanical properties of the fabrics. Predictive models of the mechanical behaviour of non-wovens in terms of their microstructure have been studied by various researchers. Komori et al. modelled the compressibility of fibrous assemblies using fibre contact theory [1]. In further work they improved their model by introducing a modified fibre contact theory [2]. The main structural parameters used in their study were the orientation function, fibre crimp and elasticity of the fibres. Patel and Warner have modelled the bending stiffness of point-bonded fabrics using the properties of the constituent fibres and the fabrics microstructure [3]. In micromechanical modelling of fibrous assemblies, the microstructural parameters form the basis of the analysis.

Therefore, it is of fundamental importance to be able to assess the microstructural parameters such as fibre orientation distribution and curl of the fibres for modelling purposes. In this study, image processing techniques have been developed to investigate the microstructural properties of fabrics made by the hydroentanglement process [4], and

The MS was received on 20 November 2001 and was accepted after revision for publication on 16 April 2002.

* Corresponding author: Wolfson School of Manufacturing and Mechanical Engineering, Loughborough University, Loughborough, Leicestershire, LE11 3TU, UK. the mechanical properties of fabric samples, such as tensile strength and modulus, have been measured.

\section{HYDROENTANGLEMENT}

Hydroentanglement is a purely mechanical bonding technology used for non-woven fabric production. It uses very fine high-speed jets of water that impact the web, penetrating into its inner planes, resulting in reorientation and entanglement of the constituent fibres. The impact of the high-speed water jets causes the fibres and fibre bundles to twist, bend and rotate around themselves and other fibres to form a series of small, interlocking entanglements [5]. The process uses successive stages of entanglement either by deploying multiple water jet stations or feeding the fabric through the machine several times. Both sides of the fabric are normally processed, either by impacting both sides of the fabric by water jets in one pass or by flipping the fabric over in alternate passes, depending on the configurations of the machine used. Thus, the consolidated fabric is bonded by friction, resulting in a soft yet relatively strong fabric.

One of the main factors that changes through the hydroentanglement process is the friction, which plays a predominant role in determining the mechanical behaviour of fabrics as reported by Hearle and Sultan [6]. The energy required at each progressive stage of the hydroentanglement process depends on the interfibre friction and the entanglement sites that have already been imparted to the fabric in 
the previous stage. Through this energy transfer, the microstructure of the fibrous assembly progressively changes and its mechanical properties are consequently improved. Web properties such as area density, fibre arrangement and structure, determined by the method used in the web production (e.g. parallel or cross-laid), and the properties of the constituent fibres such as modulus, bending stiffness, length and interfibre friction are some of the factors that determine the amount of energy needed for a desirable entanglement [7].

The main microstructural changes that occur in the fabric through the process of hydroentanglement are manifested by:

(a) a change in fibre orientation distribution through rearrangement of the fibres;

(b) a change in the apparent length of the straight segments of fibres through the curling and migration of fibres into the inner planes of the fibrous assembly.

The main microstructural parameters assessed here are therefore the fibre orientation distribution and the distribution of the straight segments of the fibres in the fabric. These parameters can be applied to estimate the level of fibre contact points and the friction between fibres, which are important parameters in the study of the fabric microstructure. The fibre orientation distribution can also be used as a measure of the anisotropy of the fabric.

The modulus and strength of a non-woven fabric directly depend not only on the physical properties of the fibres but also on the arrangement of the fibres in the fabric, e.g. the fibre orientation, the curl and the friction between the fibres at the points of the contact. These mechanical properties can relate to the microstructural properties of the non-woven fabrics.

\section{EFFECT OF PROCESSING ON THE BONDING STRENGTH AND MICROSTRUCTURE OF THE FIBRE ASSEMBLIES}

In this study, scanning electron micrographs and twodimensional Fourier and Hough transforms of the image have been used to estimate the fibre orientation distribution, and these two methods have been compared. The distribution of the length of the straight fibre segments has been found using the Hough transform (HT) of the fabric images. The mechanical properties of the fabrics (i.e. strength and modulus) have also been measured. The relationship between these microstructural variables and the mechanical properties has been analysed.

\subsection{Fibre orientation distribution using the fast Fourier transform (FFT)}

In this study, two different fabrics were used as supply webs. These webs were hydroentangled and tested to examine the effect of the hydroentanglement process on the fibre orientation distribution using the FFT method. These two webs are:

(a) web 1: 70:30 viscose-polyethylene terephthalate (PET) fibre blend, $120 \mathrm{~g} / \mathrm{m}^{2}$ cross-laid web;

(b) web 2: 70:30 viscose-PET fibre blend, $110 \mathrm{~g} / \mathrm{m}^{2}$ parallel-laid web.

The processing speed was $10 \mathrm{~m} / \mathrm{min}$. The pressure profile in different consecutive passes of the fabric through the hydroentanglement machine is given in Tables 1 and 2 respectively. The first and the second sides of the fabric were processed through four and three different passes respectively. Numbers 1.1 to 1.4 refer to the fabric samples obtained after processing the first side of the fabric, and numbers 2.1 to 2.3 correspond to the fabric samples obtained after processing the second side of the fabric.

The first test was performed on fabrics made of a 70:30 viscose-PET fibre blend, $120 \mathrm{~g} / \mathrm{m}^{2}$ cross-laid web. This web was processed under the pressure profile reported in Table 1.

Figure 1 shows scanning electron microscope (SEM) images of the tested fabric after various stages of hydroentanglement. Figure 2 shows the tensile strength and modulus of the fabric samples, measured using the EDANA standard. Details of the experimental procedure have already been reported [7].

The SEM images of fabric samples (Fig. 1) after the first side processing illustrates a much looser fibrous structure for fabric sample 1.1 than for fabric sample 1.3 (Figs $1 \mathrm{a}$ and $\mathrm{b}$ respectively). Inspection of fabric sample 2.1 (after the first pass of the second side) reveals a tightly consolidated fabric structure already achieved at this stage. In contrast to the first side samples, the difference between second side fabric structures 2.1 and 2.3 is relatively small, as shown in Figs 1c and $d$ respectively. Successive processing of the fabric increases the number of entanglement points at a gradually diminishing rate owing to increasingly tightly packed fibrous structures resisting further fibre movement. Therefore, the effect of processing of the first side of the fabric on the entanglement and the fabric microstructure is much greater. This explains the higher rate of increase in the tensile strength and modulus for the fabric samples from the first side processing compared with the second side processing (Figs 2a and b). In these figures, MD, CD and DD indicate machine direction, cross-direction and diagonal direction $\left(45^{\circ}\right.$ to $\mathrm{MD}$ and $\left.\mathrm{DD}\right)$ respectively.

In the example of a fabric made from cross-laid web, the large increase in the fabric strength in the cross-direction indicates an increase in the degree of entanglement, while

Table 1 Processing conditions using the $120 \mathrm{~g} / \mathrm{m}^{2}$ viscose-PET cross-laid web (machine speed $10 \mathrm{~m} / \mathrm{min}$ )

\begin{tabular}{llllllll}
\hline \multicolumn{2}{l}{ First side pass number } & & & \multicolumn{3}{c}{ Second side pass number } \\
\cline { 1 - 2 } \cline { 6 - 8 } & 1.1 & 1.2 & 1.4 & & 2.1 & 2.2 & 2.3 \\
\hline $60 \mathrm{bar}$ & $100 \mathrm{bar}$ & $130 \mathrm{bar}$ & $130 \mathrm{bar}$ & & $110 \mathrm{bar}$ & $130 \mathrm{bar}$ & $130 \mathrm{bar}$ \\
\hline
\end{tabular}


Table 2 Processing conditions using the $110 \mathrm{~g} / \mathrm{m}^{2}$ viscose-PET parallel-laid web (machine speed $10 \mathrm{~m} / \mathrm{min}$ )

\begin{tabular}{|c|c|c|c|c|c|c|}
\hline \multicolumn{4}{|c|}{ First side passes } & \multicolumn{3}{|c|}{ Second side passes } \\
\hline 1.1 & 1.2 & 1.3 & 1.4 & 2.1 & 2.2 & 2.3 \\
\hline 50 bar & $100 \mathrm{bar}$ & $120 \mathrm{bar}$ & $120 \mathrm{bar}$ & $100 \mathrm{bar}$ & $120 \mathrm{bar}$ & $120 \mathrm{bar}$ \\
\hline
\end{tabular}

the increase of strength in the machine direction a shows change in fibre orientation towards the machine direction. The relative number of fibres in any direction generally correlates with the strength and modulus of the fibres in that direction.

The fibre orientation distribution estimated using the Fourier transform (FT) for fabrics 1.1 and 1.3 are compared in Fig. 3a. A similar comparison is made for fabrics 2.1 and 2.3, as shown in Fig. 3b. The predominant orientation of the fibres is in the cross-direction $\left(90^{\circ}\right)$, as expected from a cross-laid web. An increase in fibre distribution in the machine direction is observed throughout the entire entanglement process. However, the change in the orientation distribution from fabric 1.1 to fabric 1.3 is more significant than the change from fabric 2.1 to fabric 2.3. In processing the first side of the fabric, since there is less initial bonding between the fibres, they can be reoriented with less effort. As the hydroentanglement process successively moves from one stage to the next, the number of entanglement points between the fibres increases progressively, hence at each stage rendering the movement and migration of fibres more difficult.

The second test was performed on fabrics made of a $70: 30$ viscose-PET fibre blend, $110 \mathrm{~g} / \mathrm{m}^{2}$ parallel web. This web was processed under the pressure profile reported in Table 2. In this case, for the parallel-laid web, most of the fibres are originally oriented in the machine direction, and therefore the modulus and strength in the machine direction are higher for all samples than those in the cross-direction. The strength and modulus of the machine direction increase significantly from fabric sample 1.1 to 1.3 and from 2.1 to 2.3, as shown in Figs $4 a$ and b, indicating gradual increase in the entanglement of the fibres. Figure 4 shows a very slight increase both in the strength and modulus of the fabric in the cross-direction, indicating little change in the direction of fibre orientation

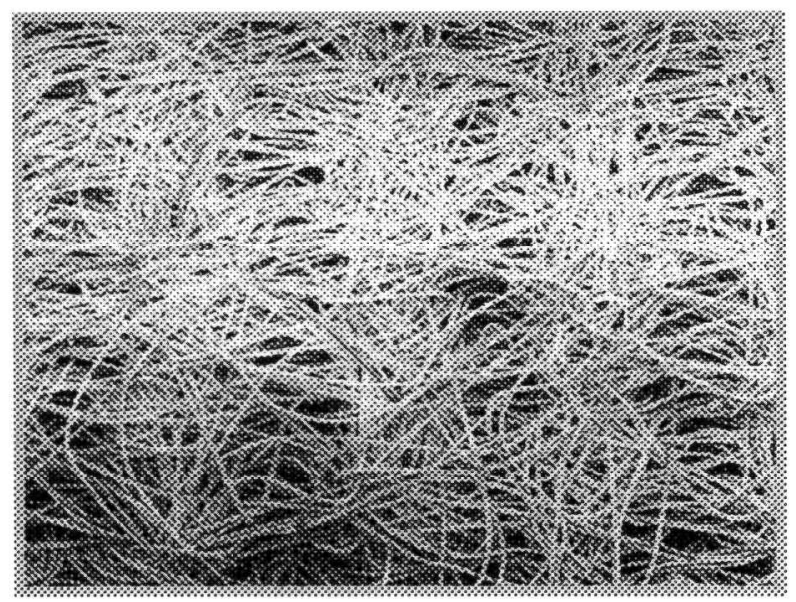

(a) First side, 1.1

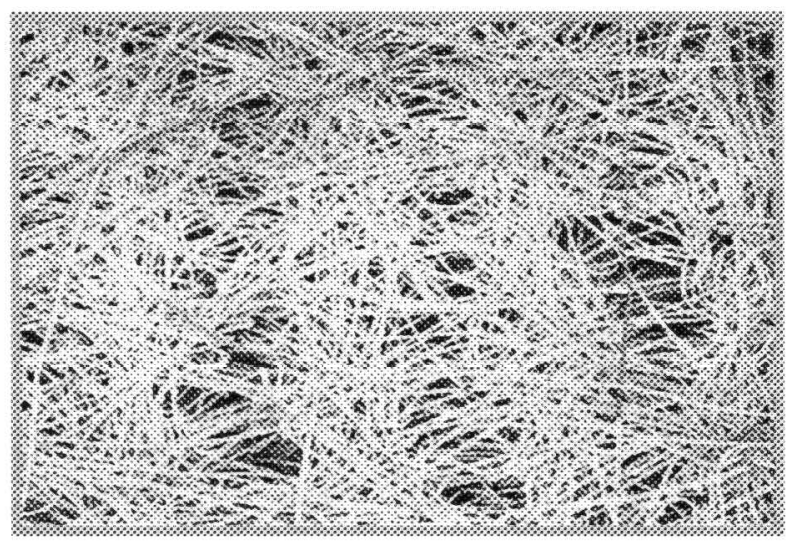

(c) Second side, 2.1

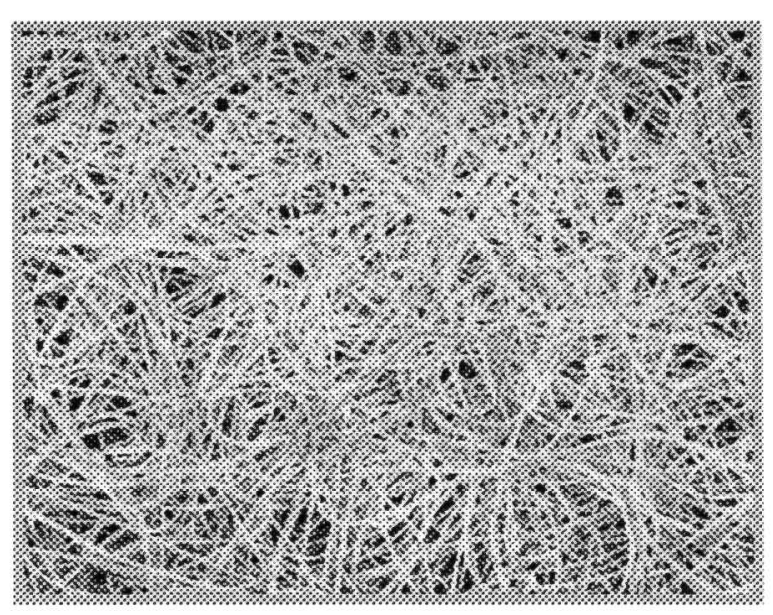

(b) First side, 1.3

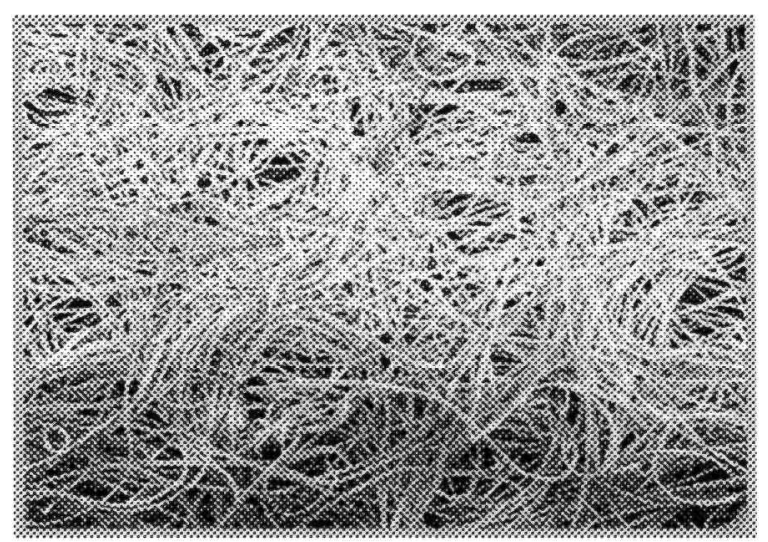

(d) Second side, 2.3

Fig. 1 SEM images of viscose-PET cross-laid fabrics (Table 1) 


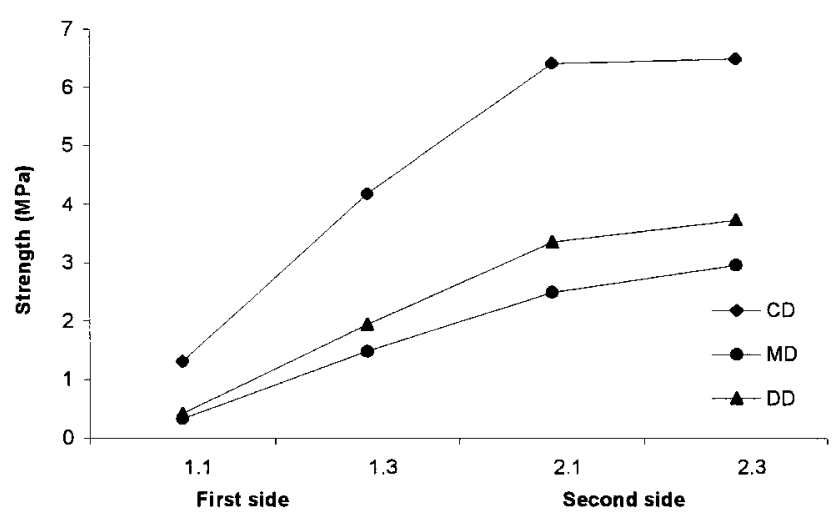

(a)

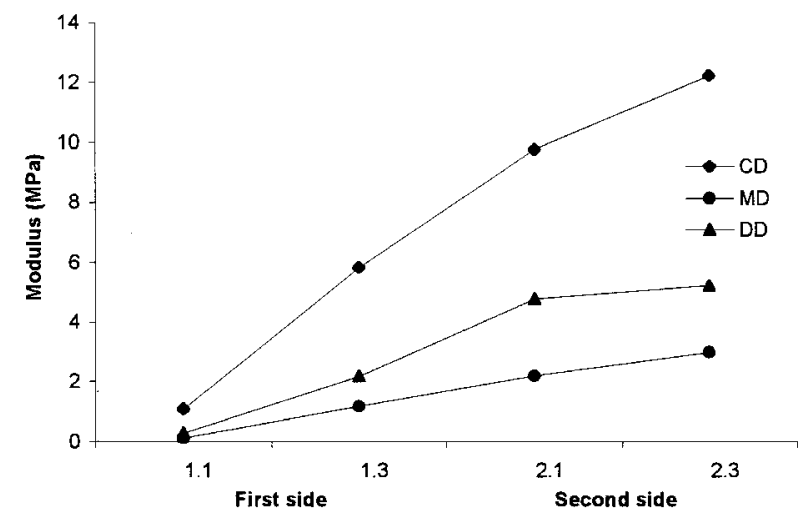

(b)

Fig. 2 (a) Tensile strength and (b) tensile modulus of viscosePET cross-laid fabrics (Table 1): CD, cross direction; $\mathrm{MD}$, machine direction; DD, diagonal direction

from the machine direction to the cross-direction owing to the consolidation of the web.

The fibre orientation distribution estimated for the fabric samples obtained after the first and third passes of the first side processing, 1.1 and 1.3 respectively, are compared in Fig. 5a. Figure 5b shows a similar comparison for the fabric samples obtained after the first and third passes of the second side processing, 2.1 and 2.3 respectively. Since the main orientation of the fibres forming the web is in the machine direction, the maxima of all the distribution curves occur at $0^{\circ}\left(180^{\circ}\right)$, i.e. the machine direction. As indicated by the results for each fabric sample, the predominant orientation of the fibres remains the machine direction, with little increase in the fibre orientation in the cross-direction. There is a greater increase in the fibre orientation in the crossdirection after the first side processing than after the second side processing, as expected.

The overall change in the fibre orientation in the crosslaid fabric is greater than that of the parallel-laid fabric. This can be concluded by comparing the results of fabrics made of cross-laid web (Fig. 3) with those of fabrics made of parallel web (Fig. 5). This is due to the nature of the hydroentanglement process in that the impinging jets

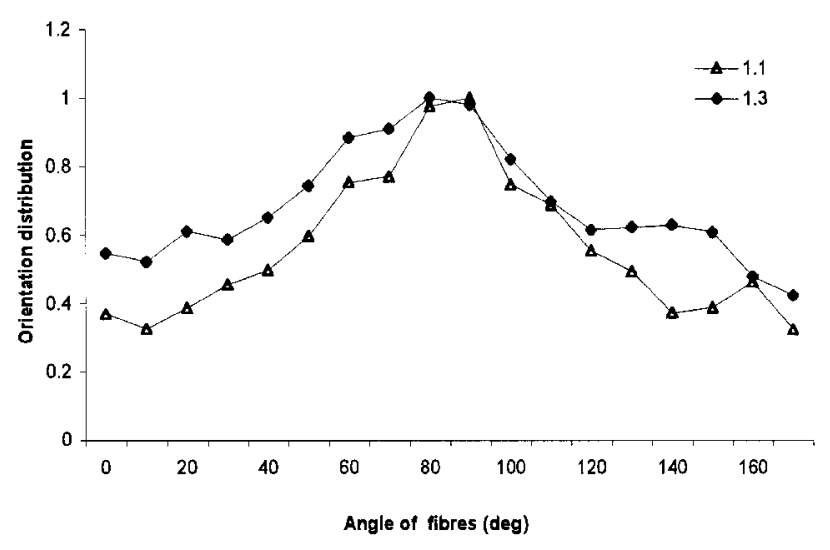

(a) First side

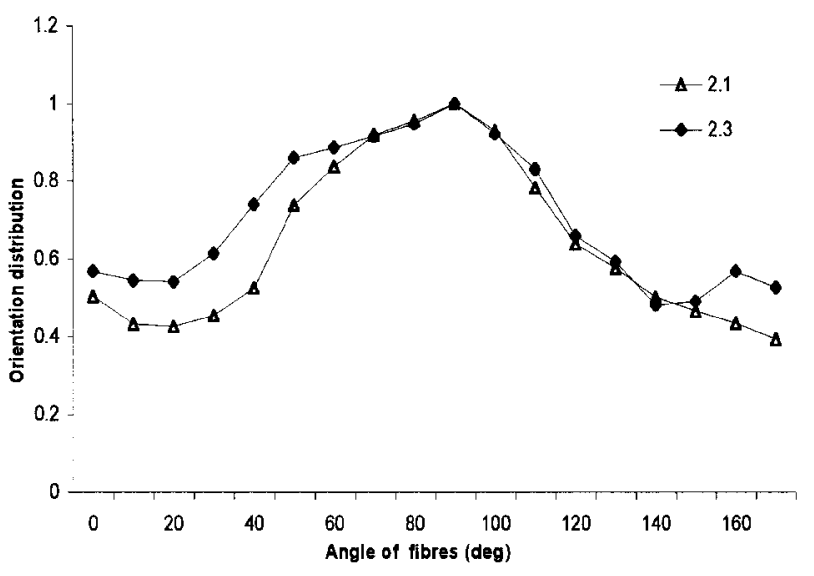

(b) Second side

Fig. 3 Fibre orientation distribution of viscose-PET cross-laid fabrics (Table 1) (a) on the first side and (b) on the second side, evaluated by the FFT method

continuously move relative to the fabric in the machine direction, dragging fibres to migrate from the cross-direction to the machine direction with more ease.

\subsection{Fibre orientation distribution using the Hough transform}

It has been shown that the fibre orientation distribution can also be estimated by the HT method [4], as the orientation of the lines is one of the direct HT results. On the other hand, in the FFT method, the orientation of the line is derived by the parameter of the rate of change in grey scale of the original image. Therefore, the HT result might be expected to be more accurate. We have carried out a number of tests to compare FFT and HT results in evaluating the fibre orientation distribution.

The first test used a fabric made of cross-laid viscosePET web (Table 1). The HT result is highly affected by the intervals chosen to pick up the local maximum and by the discretization of the Hough space. Figure 6a compares the 


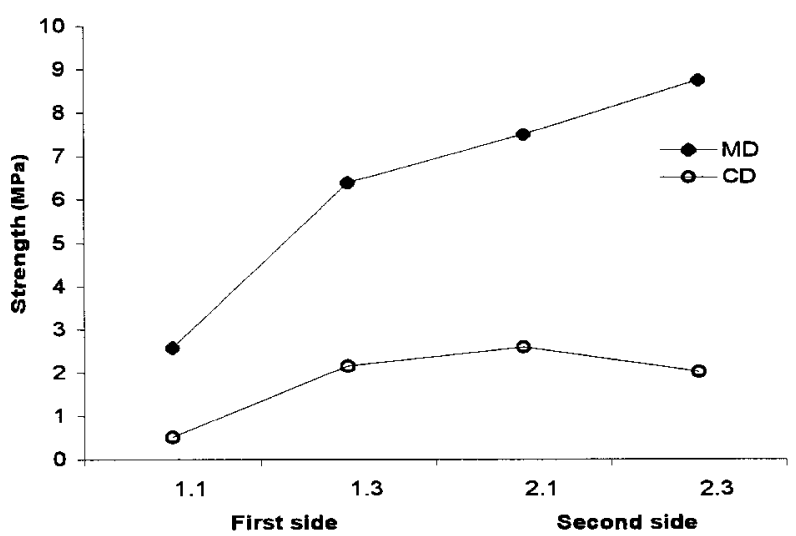

(a)

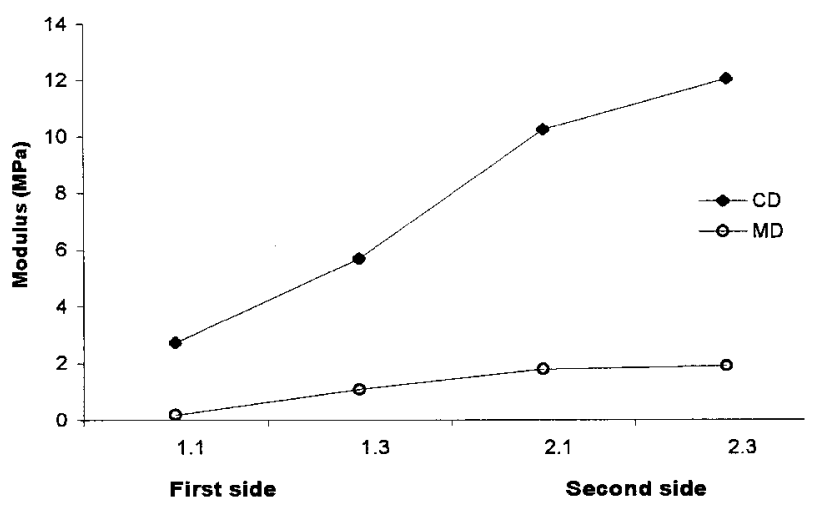

(b)

Fig. 4 (a) Tensile strength and (b) tensile modulus of viscose-PET parallel fabrics (Table 2): CD, cross direction; MD, machine direction

orientation distribution using different numbers of cells (50, 100 and 200) in Hough space. The accuracy of the method in estimating the fibre orientation distribution increases with an increasing number of cells in Hough space. On the other hand, increasing the number of cells also increases the computational time. Therefore, there is a need for a compromise between accuracy and the demand on computation time. For the purpose of the present investigation, numerous tests have shown that a 100-cell Hough space is the optimum size cell discretization for acceptable accuracy and computing time. This is discussed in more detail in Part 1 of this paper [4]. The fibre orientation distribution obtained by the FFT method and the HT method using 100-cell Hough space are compared in Fig. 6b. The good agreement between these results is evident.

The second test made a similar comparison between the fibre orientation distribution for a hydroentangled fabric produced from parallel-laid viscose-PET web (Table 2) as estimated by the FFT and HT methods (Fig. 7). The normalized results show once again a reasonable agreement between the outcome of the two methods.

The HT method was used to investigate the effect of first side and second side processing on the fibre orientation distribution. This test was performed on a viscose-PET

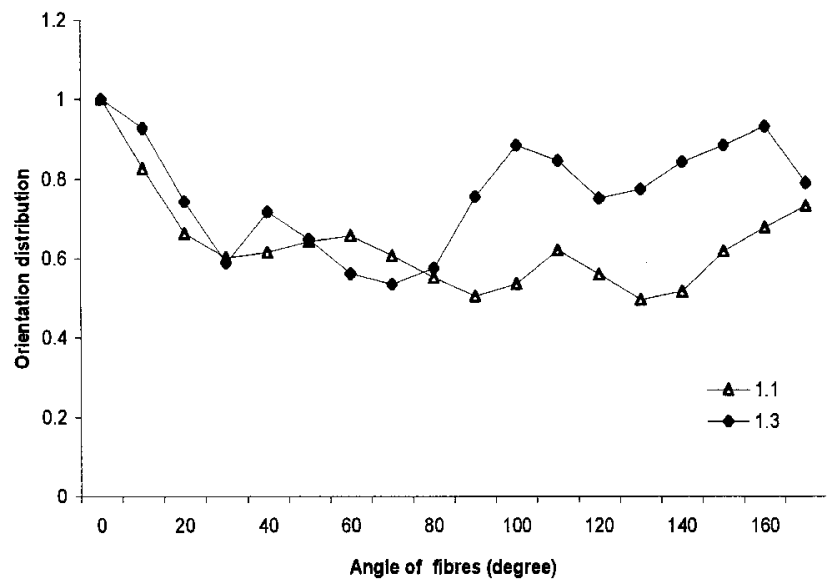

(a)

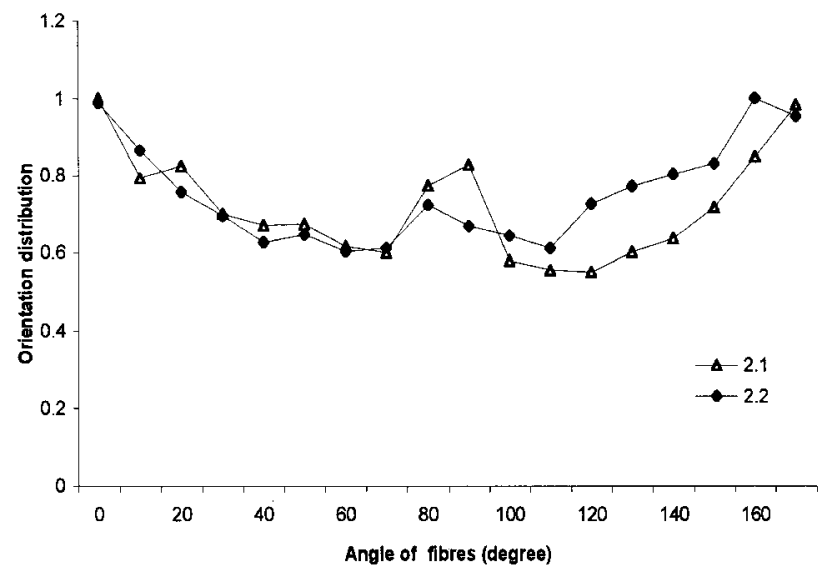

(b)

Fig. 5 Fibre orientation distribution of viscose-PET parallel fabrics (Table 2) (a) on the first side and (b) on the second side, evaluated by the FFT method

cross-laid web which was processed under the pressure profiles shown in Table 3.

The results of the HT estimation are shown in Figs 8a and $b$. The solid lines in these figures are the curves fitted to show the distribution function. The discrete points show the immediate outcome of the HT for orientation distribution. The main orientation of the fibres is in the cross-direction, as expected from a cross-laid web. Processing the fabric through more passes caused fabric 1.3 to have a more uniform distribution compared with fabric 1.1. Figure $8 \mathrm{~b}$ shows the fibre orientation distribution estimated by the HT method for fabric samples after pass 2.1 and 2.3. The orientation distributions of these two fabric samples are much more similar. The fibre orientation distribution estimated by the HT method, gives similar results to that estimated by the FFT method, presented earlier.

In this final test, a comparison of the FFT and HT techniques was made using a fabric sample with more curled fibres. This fabric was made of a viscose-PET cross-laid web, which was processed under the pressure profile reported in Table 4. Under this high pressure, much 


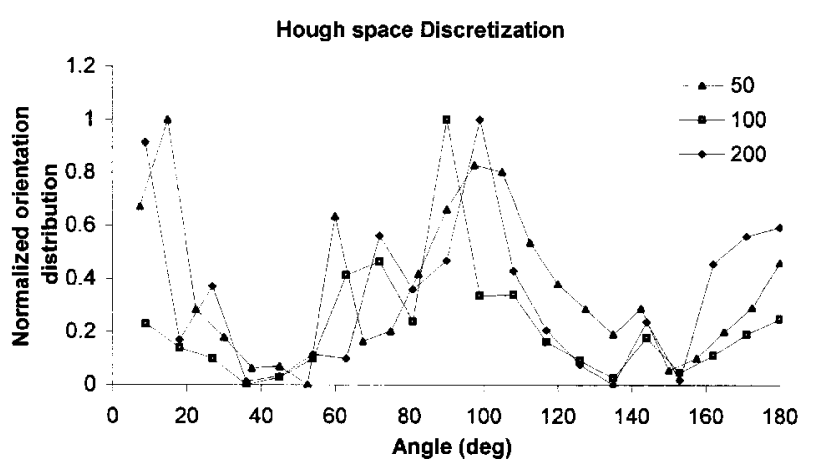

(a)

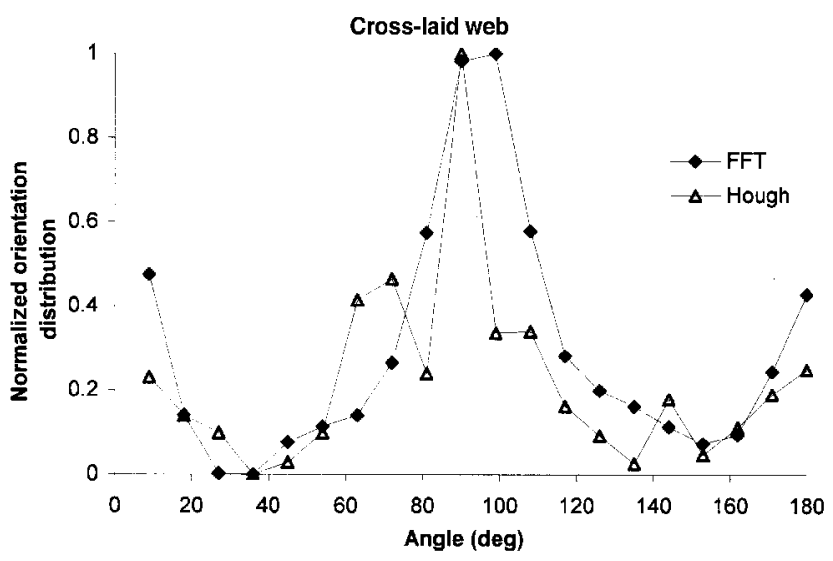

(b)

Fig. 6 Effect of Hough space discretization on the fibre orientation distribution evaluated for viscose-PET cross-laid fabric (Table 1) and (b) comparison of the FFT and HT results for the fibre orientation distribution of the fabric

more curled fibres are produced by the hydroentanglement process compared with the fabric made under previous pressure profiles (Tables 1 to 3 ).

Since the HT works on the basis of recognition of straight lines, in this method the lines representing the fibres are

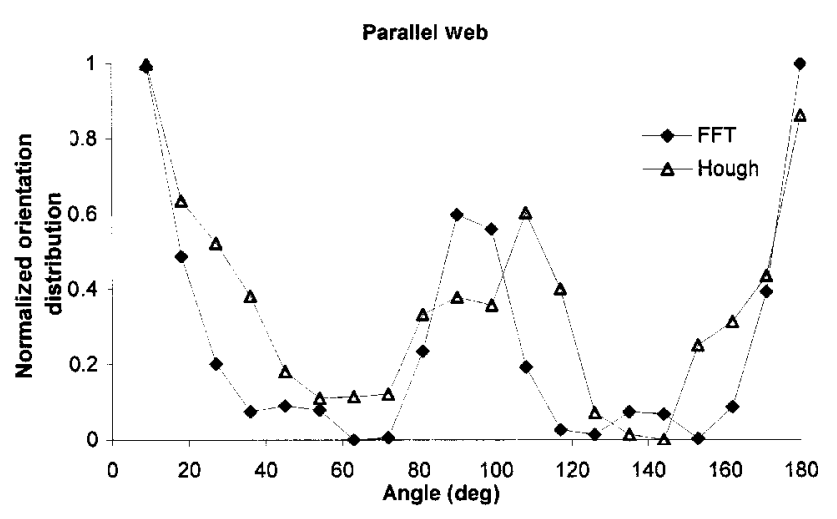

Fig. 7 Comparison of the FFT and HT results for the fibre orientation distribution of viscose-PET parallel fabric (Table 2)
Table 3 Processing conditions using the $120 \mathrm{~g} / \mathrm{m}^{2}$ viscose-PET cross-laid web (machine speed $10 \mathrm{~m} / \mathrm{min}$ )

\begin{tabular}{llllllll}
\hline First side passes & & & & \multicolumn{3}{c}{ Second side passes } \\
\cline { 1 - 3 } \cline { 6 - 8 } 1.1 & 1.2 & 1.3 & 1.4 & & 2.1 & 2.2 & 2.3 \\
\hline $50 \mathrm{bar}$ & $70 \mathrm{bar}$ & $100 \mathrm{bar}$ & $100 \mathrm{bar}$ & & $80 \mathrm{bar}$ & $100 \mathrm{bar}$ & $100 \mathrm{bar}$ \\
\hline
\end{tabular}

segmented into straight-line parts. The FFT method does not have this restriction. Because of this property, the outcome of the HT method is only comparable with the FFT method when it is applied to skeletonized images. Figure 9 compares the fibre orientation distributions evaluated by the HT method using a skeletonized image and by the FFT method using both skeletonized and binarized images. While the FFT results using skeletonized images are in reasonable agreement with the HT results, the FFT estimation of the binarized images is considerably different. This can be attributed to the fact that the curl of the fibres causes the skeletonized image to be considerably different to the binarized images. This shows that, as the curl of the fibre in a fabric increases, the accuracy of the HT method in estimating fibre orientation distribution reduces.

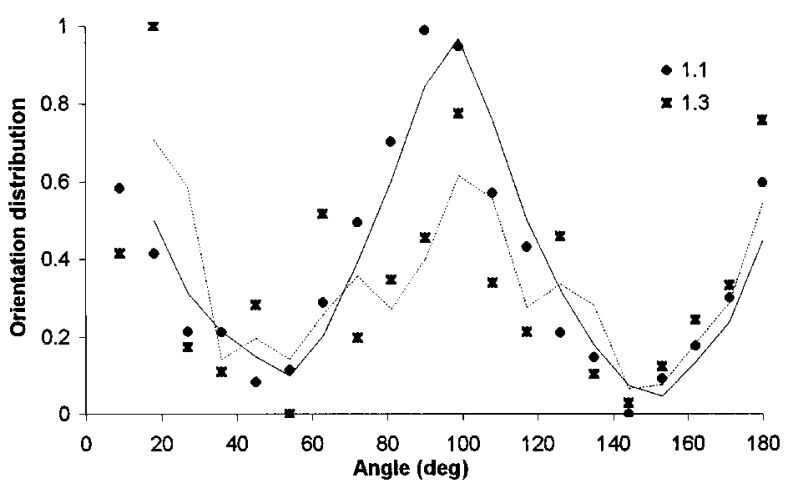

(a)

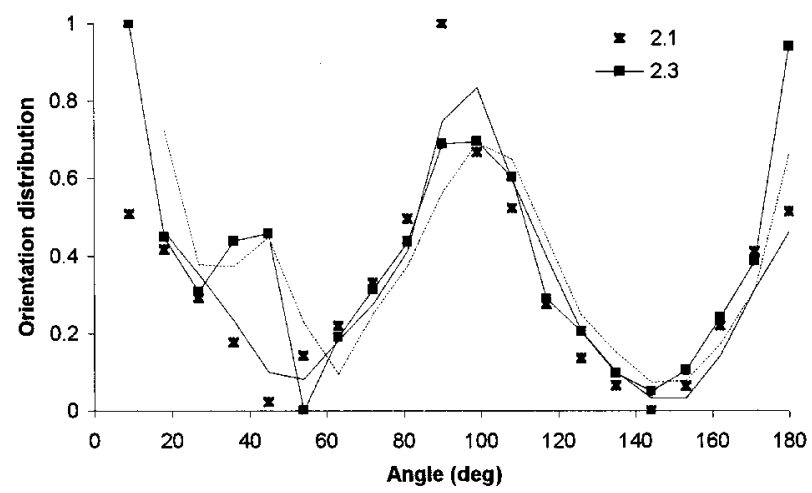

(b)

Fig. 8 Fibre orientation distribution of viscose-PET cross-laid fabric (Table 3) (a) on the first side and (b) on the second side, evaluated by the HT method 
Table 4 Processing conditions using the $120 \mathrm{~g} / \mathrm{m}^{2}$ viscose-PET cross-laid web (machine speed $10 \mathrm{~m} / \mathrm{min}$ )

\begin{tabular}{llllllll}
\hline \multicolumn{3}{l}{ First side passes } & & & & \multicolumn{3}{c}{ Second side passes } \\
\cline { 1 - 3 } \cline { 5 - 7 } 1.1 & 1.2 & 1.3 & 1.4 & & 2.1 & 2.2 & 2.3 \\
\hline $50 \mathrm{bar}$ & $120 \mathrm{bar}$ & $170 \mathrm{bar}$ & $170 \mathrm{bar}$ & & $130 \mathrm{bar}$ & $170 \mathrm{bar}$ & $170 \mathrm{bar}$ \\
\hline
\end{tabular}

\subsection{Distribution of the length of straight segments of fibres}

The distribution of the length of straight segments of fibres in a fabric made from a $120 \mathrm{~g} / \mathrm{m}^{2} 70: 30$ viscose-PET blend cross-laid web (shown in Table 1) was evaluated by applying HT analysis. Images of the fabrics after several passes were tested. The results of the HT and post-processing described earlier are shown in Figs 10a and b. In these figures, the fibre

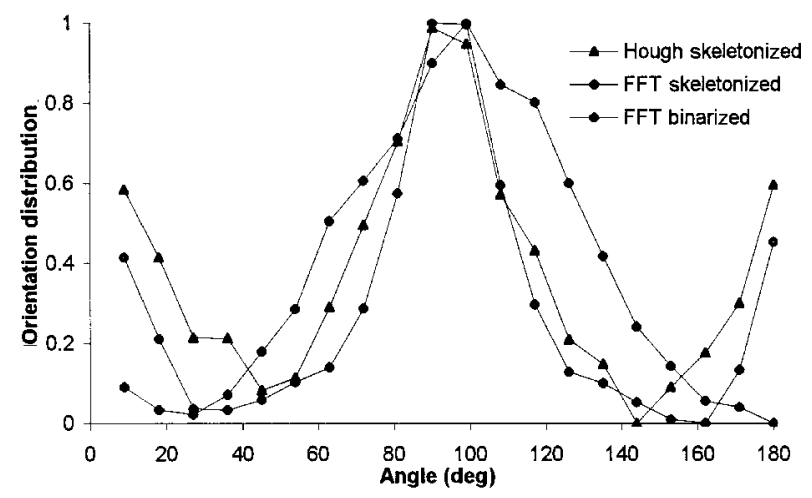

Fig. 9 Comparison of the results of FFT (skeletonized and binarized) and HT (skeletonized) for the fibre orientation distribution of viscose-PET cross-laid fabric with curled fibres (Table 4)

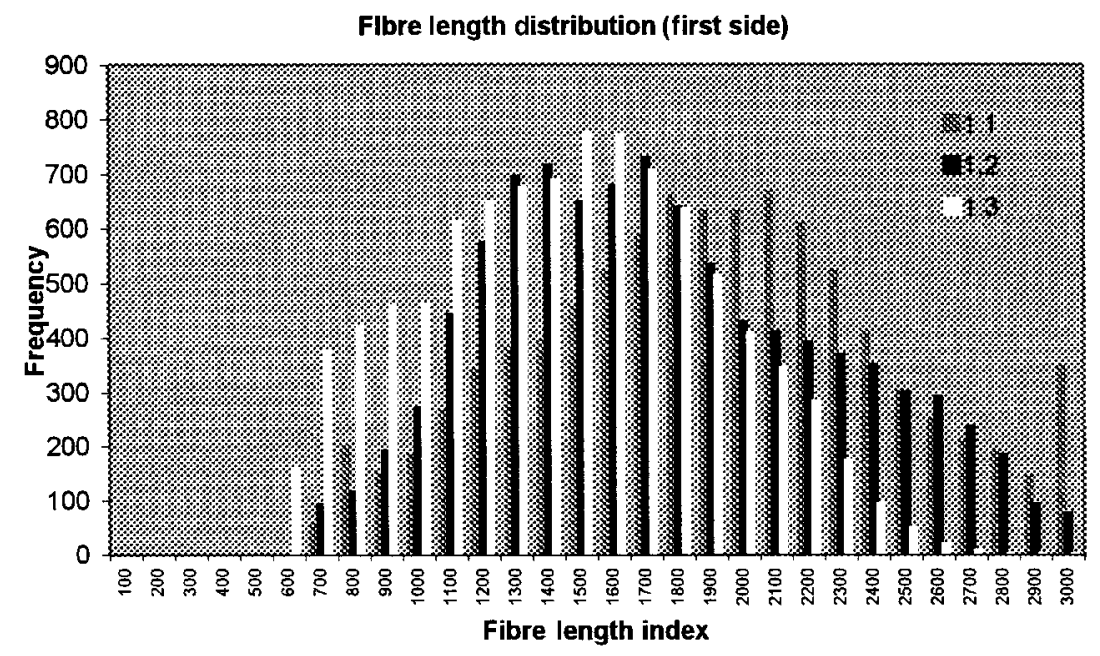

(a)

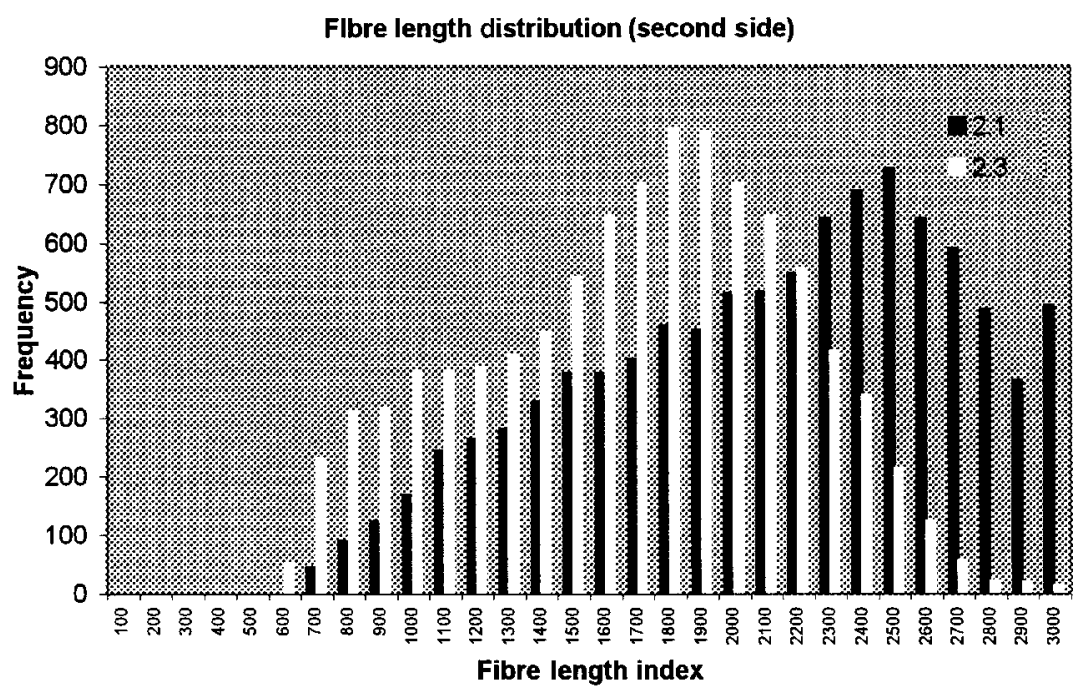

(b)

Fig. 10 Fibre length distribution of viscose-PET cross-laid fabrics (Table 1) (a) on the first side and (b) on the second side, evaluated by the HT method 
length index is used, as the values on the horizontal axis do not show the exact length of the fibres but are proportional to the length of the fibres. Figure 10a shows the fibre length distribution in fabric samples 1.1, 1.2 and 1.3, all first side processing. As the length distribution for these fibres verifies, the length of the straight segments of the fibres decreases from sample 1.1 to sample 1.2 to sample 1.3 , as indicated by the fibre length indices. The results of the second side for fabrics 2.1 and 2.3 are presented in Fig. $10 \mathrm{~b}$. A similar trend of change is also observed, as the length of the straight segments of the fibres decreases from fabric sample 2.1 to sample 2.3 .

It should be noted that the actual length of the fibres does not change through the process. However, as the entanglement process proceeds, fibres are moved from one plane to another, and at the same time they bend and curl. This results in a reduction in the length of the straight segments of fibres, as detected by HT analysis. Therefore, the most frequent fibre length in each distribution curve correlates with the degree of bonding achieved through entanglement. The lower the most frequent fibre length, the higher is the level of entanglement. The fibre length distribution can therefore be used as a measure of the fibre entanglement in the hydroentangling process.

\section{CONCLUSIONS}

The image processing techniques developed in Part 1 using FFT and HT methods in conjunction with SEM images have successfully been used in evaluating microstructural changes in hydroentangled fabrics, such as fibre orientation and fibre length distribution. Mechanical fabric properties such as strength and modulus also support the validity of the above techniques, which can conveniently be used to estimate the degree of entanglement in hydroentangled fabrics.
The results of the FFT and HT methods have been compared. It has been shown that the results of the skeletonized images by the FFT and HT techniques are in very good agreement. The outcome of the HT analysis is highly affected by the Hough space discretization. This needs to be optimized for a given fabric sample.

The image processing techniques developed have been successfully tested for fabrics up to $120 \mathrm{~g} / \mathrm{m}^{2}$. This shows the applicability of the methods for heavy and thick fabrics. In cases where the thickness of the fabric is higher than the depth of field of the SEM, a two-dimensional analysis would be less accurate. In these cases, a three-dimensional analysis including the depth of the fabric is required.

\section{REFERENCES}

1 Komori, T., Itoh, M. and Takaku, A. A model analysis of the compressibility of fibre assemblies. Textile Res. J., 1992, 62(10), 567-574.

2 Komori, T., Itoh, M. and Takaku, A. Analysing the compressibility of a random fibre mass based on the modified theory of fibre contact. Textile Res. J., 1997, 67(3), 204-210.

3 Patel, S. and Warner, S. Modelling the bending stiffness of the point-bonded nonwoven fabrics. Textile Res. J., 1994, 64(9), 507-513.

4 Ghassemieh, E., Acar, M. and Versteeg, H. K. Microstructural analysis of non-woven fabrics using scanning electron microscopy and image processing. Part 1: development and verification of the methods. Proc. Instn. Mech. Engrs, Part L: J. Materials: Design and Applications, 2002, 216, 199-207.

5 Ikeda, M., Ishikawa, T. and Shim, T. US Pat. 4, 146, 663, 1998. 6 Hearle, J. W. S. and Sultan, M. A. I. J. Textile Inst., 1968, 70, 19.

7 Ghassemieh, E., Acar, M. and Versteeg, H. K. Improvement of the efficiency of energy transfer in the hydroentanglement process. Composite Sci. and Technol., 2001, 61(12). 